\title{
Lipid-Head-Polymer-Tail Chimeric Nanovesicles
}

\author{
Kan Hu, ${ }^{[a]}$ Aoyuan Cheng, ${ }^{[a]}$ Dingcheng Zhou, ${ }^{[a]}$ Yi Luo, ${ }^{[a]}$ and Guoqing Zhang ${ }^{*[a]}$
}

Dedicated to Prof. Cassandra L. Fraser for her contribution to the field of ring-opening polymerzation.

[a] Dr. K. Hu, Mr. A. Cheng, Mr. D. Zhou, Prof. Y. Luo and Prof. G. Zhang

Hefei National Laboratory for Physical Sciences at the Microscale

University of Science and Technology of China

Hefei, 230026, China

E-mail: gzhang@ustc.edu.cn

Supporting information for this article is given via a link at the end of the document.((Please delete this text if not appropriate))

\begin{abstract}
Lipid nanovesicles (LNVs) and polymer nanovesicles (PNVs), also known as liposomes and polymersomes, are becoming increasingly vital in global health. One recent example is the widely distributed mRNA Covid-19 vaccines. However, the two major classes of nanovesicles both exhibit their own issues that significantly limit potential applications. Here, by covalently attaching a naturally occurring phosphate "lipid head" and a synthetic polylactide "polymer tail" via facile ring-opening polymerization on a 500-gram scale, a type of "chimeric" nanovesicles (CNVs) can be easily produced. Compared to LNVs, the reported CNVs exhibit reduced permeability for small and large molecules; on the other hand, the CNVs are less hydrophobic and exhibit enhanced tolerance toward proteins in buffer solutions without the need for hydrophilic polymeric corona such as poly(ethylene glycol), in contrast to conventional PNVs. The proof-ofconcept in vitro delivery experiments using hydrophilic solutions of fluorescein-PEG, rhodamine-PEG, and anti-cancer drug doxorubicin demonstrate that these CNVs, as a structurally diverse class of nanomaterials, are highly promising as alternative carriers for therapeutic molecules in translational nanomedicine.
\end{abstract}

\section{Introduction}

Nanovesicles are an important class of biomaterials extensively used for encapsulation and administration of small molecular drugs ${ }^{[1]}$ as well as therapeutic biomacromolcules, ${ }^{[2]}$ due to multiple advantages such as reduced toxicity, long circulation time, targeted delivery, and controlled release..$^{[3]}$ Their vital role in modern medicine is further accentuated in light of the recent Covid-19 pandemic, where two major mRNA-vaccination technologies ${ }^{[4]}$ were developed from LNVs. Based on the molecular structure of the main ingredient, there are currently two major types of nanovesicle-forming materials: lipids and polymers, corresponding to LNVs (liposomes) ${ }^{[5]}$ and PNVs (polymersomes), ${ }^{[6]}$ respectively, after fabrication. Although both systems share the abovementioned advantages, LNVs suffer from problems of instability and leakage, ${ }^{[7]}$ while PNVs have in vivo safety concerns and, particularly, struggle with scalability issues, a major reason for PVNs to have "lost in translation" in the past twenty-five years. ${ }^{[8]} \mathrm{A}$ highly simplistic generalization is that LNVs rely on the delicate balance between charges and hydrophobic alkyl chains, while PNVs largely depend on the intricate interplay among different segments of a block copolymer. Therefore, the natural question to ask is whether it is possible to integrate features from the two systems in one single component, e.g., a synthetic polymer tail with a charged lipid head. A predictable outcome is that the molecule still has the tendency to form a liposome-like structure in an aqueous environment, but since the interchain interaction can be strengthened from merely entropic (hydrophobic interaction between aliphatic chains) to a combination of enthalpic and entropic by introducing a longer and more polar polymer tail, the nanovesicles thus formed could potentially gain extra stability. Furthermore, if a biocompatible and biodegradable polymer chain is employed, it also solves the problem of poor degradation common for PNVs. ${ }^{[1 c, 9]}$ Nonetheless, the downside of the system is also obvious: the statistical distribution of chain lengths could lead to non-uniformed vesicle dimensions.

Here, with the integrated design principle, we show that a chimeric nanovesicle (CNV, Scheme 1) system can be created from $100 \%$ bio-derived molecular building blocks using $L-\alpha$ glycerylphosphorylcholine ( $\alpha \mathrm{GPC}$ ) from hydrolyzed soy lipids as the initiator and L-lactide from corn fermentation as the monomer. The CNV system exhibits high vesicle-forming efficiency via an appropriate fabrication method, characterized by scanning and transmission electron microscopy (SEM and TEM) techniques. The proof-of-concepts cargo delivery of hydrophilic fluorescent and drug molecules into HeLa cells is also demonstrated. Finally, since the end-functionalized PLA is essentially a homopolymer instead of di- or triblock copolymers, synthesis in large quantities can be easily achieved (e.g., 500-g scale in the laboratory), which gives the system further potential in practical applications in the foreseeable future.

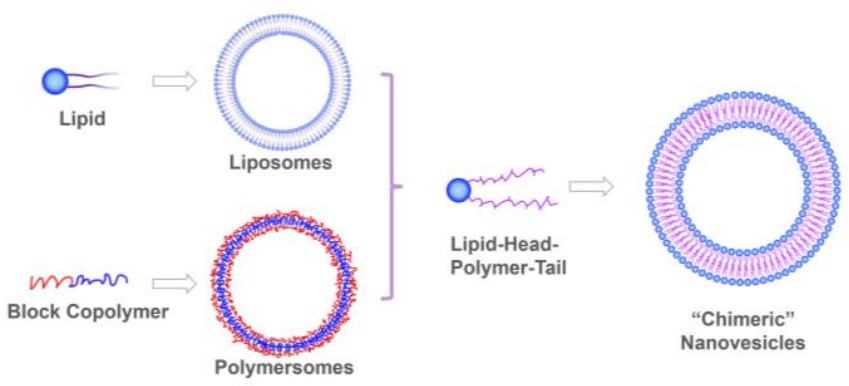

Scheme 1. Schematic illustration of incorporation of liposome and polymersome features into "chimeric" nanovesicles fabricated from a lipidhead-polymer-tail structure.

\section{Results and Discussion}

The ring-opening polymerization (ROP) of L-lactide initiated by alcohols in the presence of a Lewis acid catalyst has been 


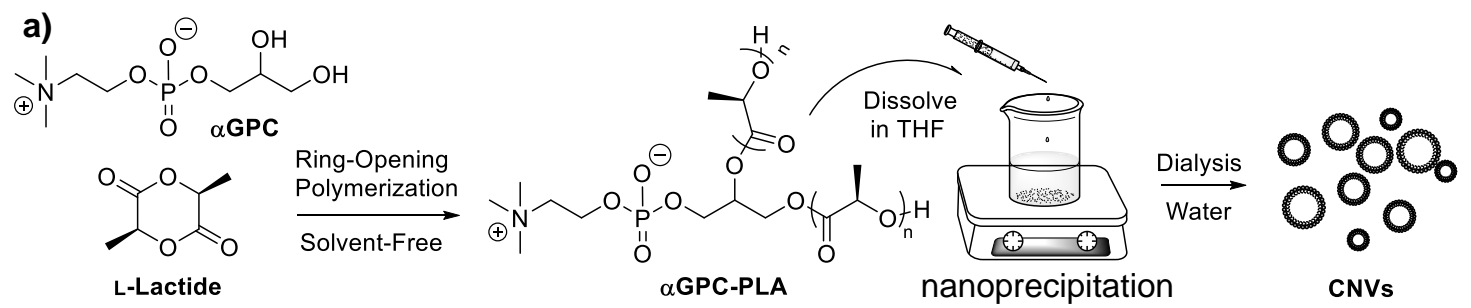

b)

$\begin{array}{cccc}\begin{array}{c}\text { CVNs } \\ \begin{array}{c}\text { Fabrication } \\ \text { Method }\end{array}\end{array} & \begin{array}{c}\alpha \mathrm{GPC} / \\ \text { Lactide } \\ \text { (CNV ID) }\end{array} & \begin{array}{c}\text { Average } \\ \text { Diameter } \\ \text { (nm) }\end{array} & \text { PDI } \\ \text { Hydration } & 1 / 30(\mathbf{H 1}) & 240.6 & 0.209 \\ & 1 / 50(\mathbf{H} 2) & 265.0 & 0.192 \\ \begin{array}{c}\text { Nano- } \\ \text { precipitation }\end{array} & 1 / 30(\mathbf{N 1}) & 229.5 & 0.182 \\ \text { Dialysis } & 1 / 50(\mathbf{N} 2) & 116.0 & 0.205 \\ & 1 / 30(\mathbf{D} 1) & 1420 & 0.348 \\ & 1 / 50(\mathbf{D} 2) & 1095 & 0.296\end{array}$
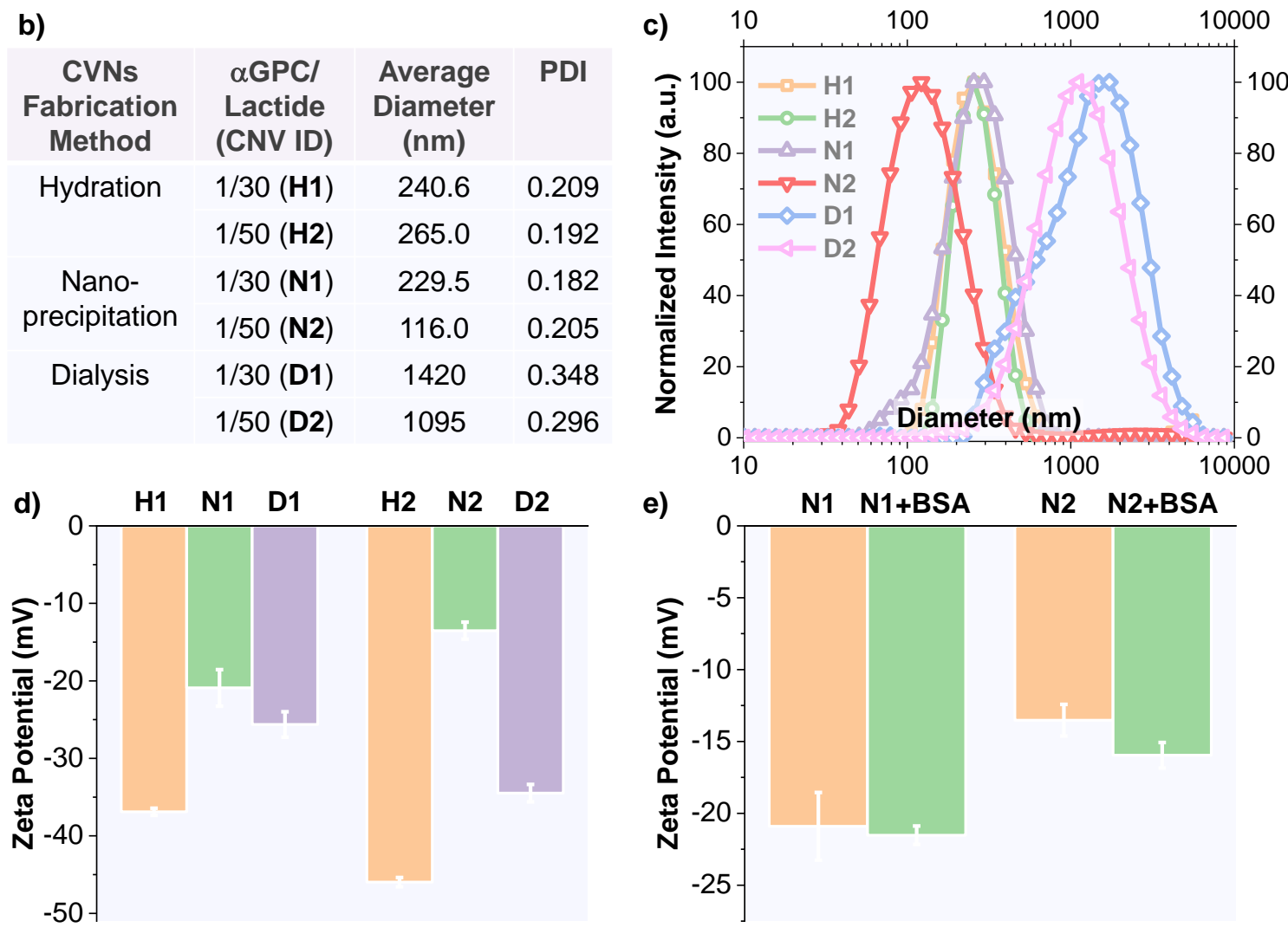

Figure 1. (a) Chemical structures of the zwitterionic initiator $\alpha \mathrm{GPC}$, the monomer L-lactide, and the resulting polymer $\alpha \mathrm{GPC}$-PLA, which can subsequently be fabricated into CNVs via an exemplar nanoprecipitation method. (b) Average sizes (diameter) and polydispersity indices (PDIs) of CNVs prepared from three different methods (hydration, $\mathbf{H} 1$ and $\mathbf{H 2}$; nanoprecipitation, N1 and N2; dialysis, D1 and D2) with $\alpha$ GPC-PLA polymers synthesized from designated initiatorto-monomer ratios (1:30 or 1:50) corresponding to MWs of 3,700 and 6,800 Da ( $\left.{ }^{1} \mathrm{H}-\mathrm{NMR}\right)$, respectively. (c) Size distributions of CNVs from dynamic lightscattering measurements. (d) Zeta potentials of CNVs prepared from different methods. (e) Change of zeta potentials for CNVs N1 and N2 after incubation with BSA in PBS at $37^{\circ} \mathrm{C}$ for $4 \mathrm{~h}$.

extensively used to synthesize end-functionalized homo- and copolymers. ${ }^{[10]}$ The solvent-free ROP could be easily used to synthesize $\alpha$ GPC-PLA at a 500-g scale in the laboratory. In this case, the $\alpha \mathrm{GPC}$ initiator (1 eq.) is dissolved in the L-lactide melt (30 or 50 eq.) at $130{ }^{\circ} \mathrm{C}$ in a $2-\mathrm{L}$ Schlenk flask sealed under nitrogen using $\mathrm{Sn}(\mathrm{Oct})_{2}$ ( 0.12 or 0.2 eq.) as the catalyst (Figure 1a). The heated liquid started to become viscous after $3 \mathrm{~h}$ and the flask was heated for another $21 \mathrm{~h}$ before it was removed from the heat bath. The presumable product, $L-\alpha$ glycerylphosphorylcholine bispolylactide ( $\alpha$ GPC-PLA), was purified by precipitation $(2 \times)$ in ethanol and in diethyl ether $(1 \times)$ from the $\mathrm{CH}_{2} \mathrm{Cl}_{2}$ solution at room temperature as a fluffy white powder. To verify that the initiator is indeed covalently attached, the ${ }^{1} \mathrm{H}-\mathrm{NMR}$ spectra were compared, where the $-\mathrm{CH}$ - and $-\mathrm{CH}_{2}-$ protons on the glyceryl group of the $\alpha \mathrm{GPC}$ initiator exhibit chemical shifts values ranging between $\delta=\sim 3.6-3.8$ ppm (Figure
S1, Supporting Information, SI), however, the esterification of the two hydroxy groups leads to a significant downfield shift $(\delta=\sim 4.3$ $4.5 \mathrm{ppm}$, Figures S2-S3) presumably due to the electronwithdrawing effects after the ROP reaction. The polymer molecular weight (MW) of $\alpha \mathrm{GPC}-\mathrm{PLA}$ is calculated by comparing integrated areas of characteristic peaks belonging to the $\alpha \mathrm{GPC}$ $N$-methyl protons ( $\delta=3.33 \mathrm{ppm}$, Figure S1-S2,) and PLA methide $(\delta=5.15 \mathrm{ppm})$ protons, respectively. For the 1:30 (1:50) ROP, the MW is found to be $3,700(6,800)$ Da with an average of $24(45)$ repeating ester groups on each chain attached to the glycerol moiety. The gel-permeation chromatography (GPC) results (Figure S5) indicate that the number (weight) averaged MWs are $5,330(7,190)$ and $69,90(10,340)$ Da for the $1: 30$ and 1:50 $\alpha \mathrm{GPC}$ PLA polymers, respectively. And the PDI calculated are 1.35 and 1.48 for $1: 30$ and 1:50 $\alpha \mathrm{GPC}-\mathrm{PLA} \mathrm{A}_{2}$ respectively. The discrepancy 

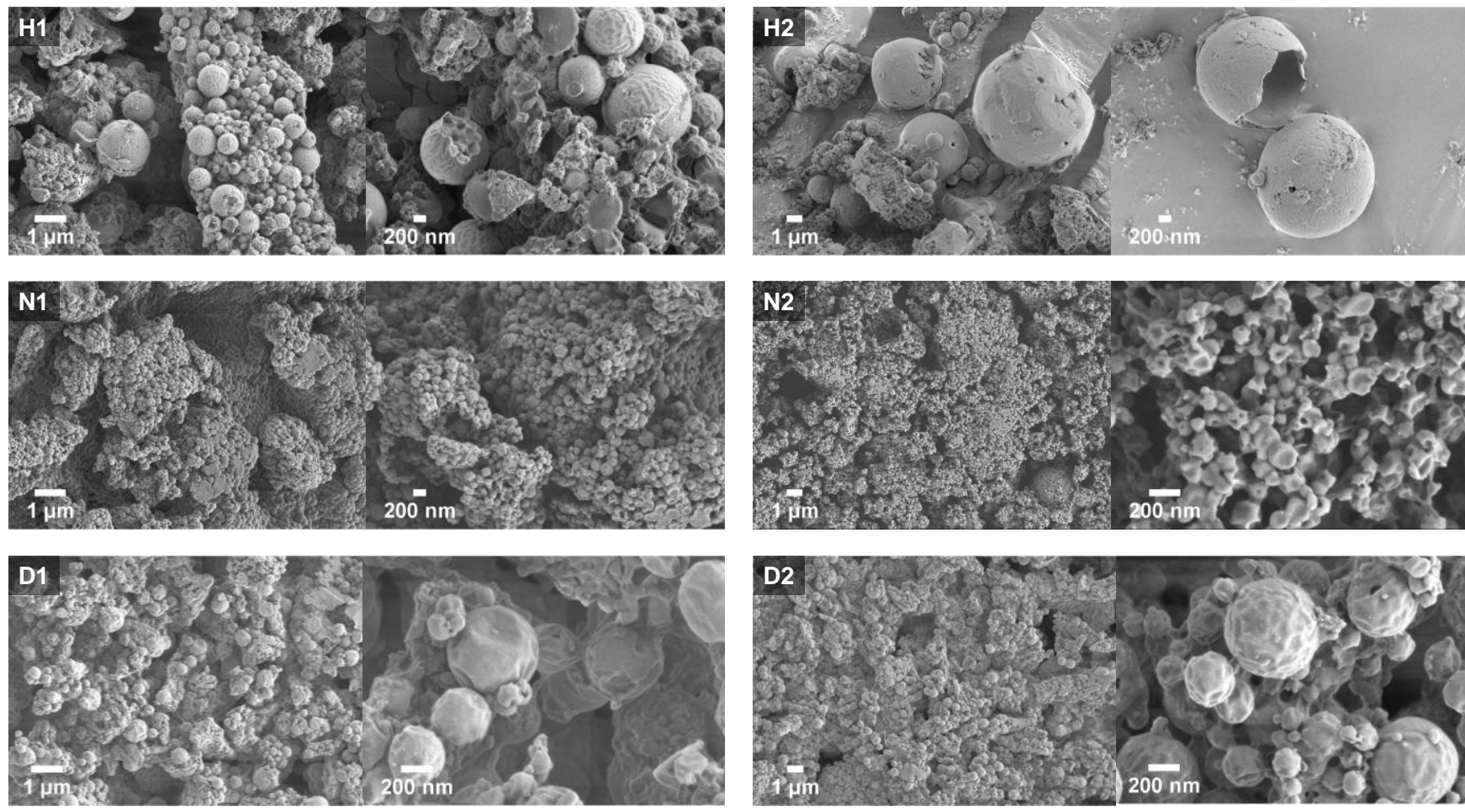

Figure 2. Scanning electron microscopy (SEM) images of free-dried CNVs prepared from different methods (hydration, H1 and H2; nanoprecipitation, N1 and N2; dialysis, D1 and D2).

in $\mathrm{MW}$ between the ${ }^{1} \mathrm{H}-\mathrm{NMR}$ and GPC methods has been previously noted for both linear and star-shaped PLA systems. ${ }^{[11]}$

To prepare vesicles, three different methods each using $\alpha \mathrm{GPC}$ PLA samples of two different MWs (Figure 1b) were employed according to literature, including hydration (H1, H2), ${ }^{[12]}$ nanoprecipitation (N1, N2), ${ }^{[13]}$ and dialysis (D1, D2), ${ }^{[14]}$ which resulted in CNVs with different sizes and morphologies potentially useful for different types of applications. The hydration method used vacuum-dried $\alpha$ GPC-PLA thin films $(20 \mathrm{mg}$ ) on the bottom of a flask, to which water $(100 \mathrm{~mL})$ was added under sonication to yield CNVs. For the nanoprecipitation method which usually produces more uniformed nanoparticles, $\alpha$ GPC-PLA $(20 \mathrm{mg})$ was first dissolved in THF (10 mL), followed by solvent displacement by dropwise addition of the THF solution into water $(100 \mathrm{~mL})$, using an automated syringe pump under vigorous stirring. The THF solvent could then be removed by stirring for overnight. The final dialysis method is usually used to prepare giant vesicles (> $10 \mu \mathrm{m})$ : the $\alpha$ GPC-PLA THF solution $(0.2 \mathrm{mg} / \mathrm{mL})$ was transferred into a dialysis bag (MWCO $1 \mathrm{kDa}$ ) and was dialyzed against ultrapure-water $(2 \mathrm{~L})$ for $48 \mathrm{~h}$. The average CNVs sizes in diameter obtained from dynamic light scattering (DLS) are presented in Figure 1b, where both hydration and nanoprecipitation afford CNVs in the $116-265 \mathrm{~nm}$ range with a PDI around 0.2 , whereas dialysis gives $\mu \mathrm{m}-\mathrm{CNV}$ s with a much larger PDI. Specifically, nanoprecipitation prepared with an initiator-tomonomer ratio of 1:50 $\left(M_{n}=6,800 \mathrm{Da}\right)$ yields the smallest CNVs. Figure 1c shows the size distribution of the six CNV samples, where the high-MW polymer is consistently favorable for smaller sizes and better uniformity. The zeta potential of each CNV system was measured, where negative values were found for all of them (Figure 1d), with the least negative being $\mathbf{N} 2(-13.5 \mathrm{mV})$ and the most negative being $\mathbf{H} 2(-46 \mathrm{mV})$. When incubated with bovine serum albumin (BSA) in phosphate-buffered saline (PBS) solution at $37^{\circ} \mathrm{C}$ for $4 \mathrm{~h}$, the zeta potential values do not seem to be appreciably influenced (Figure 1e), suggesting lack of strong interactions between the CNVs and BSA.

Next, the morphologies of these CNVs prepared from different methods were freeze-dried and characterized with scanning/transmission electron microscopy (SEM/TEM) and fluorescence microscopy (FLM) methods (Figures 2 and 3). In general, the microscopy results are consistent with the DLS measurement, that nanoprecipitation yields the smallest and most uniform CNVs, while the dialysis method could result in vesicles sizable enough to allow for FLM imaging. Although the SEM images do indicate that these CNVs have hollow interiors from water sublimation-caused collapses and friction-induced broken pieces (zoomed images of Figure 2) during sample preparation, TEM and FLM were also used to investigate the internal structures of these CNVs. In Figure 3, smaller CNVs fabricated from hydration and nanoprecipitation were examined with TEM, where the hollow spherical membranes with a thickness of $<\sim 25$ $\mathrm{nm}$ could be well-resolved. For the much larger vesicles from the dialysis method, FLM reveals that the CNVs membrane is hydrophobic, and the core is filled with aqueous solutions determined from the Sudan and fluorescein (aq.) dyes staining, respectively. Given that the CNVs fabricated from nanoprecipitation (N1 and N2) exhibit the smallest hydrodynamic diameters, the least negative zeta potentials, and the most uniform morphologies, cellular uptake experiments were performed with $\mathbf{N} 1$ and $\mathbf{N} 2$. To be biocompatible, their stabilities in PBS solution with or without the presence of BSA were examined during the course of 10 days (Figure 4a). Compared to CNVs prepared in ultrapure-water, direct nanoprecipitation in PBS results in slightly increased vesicle sizes possibly due to the interactions between the zwitterions and ions in the PBS. 


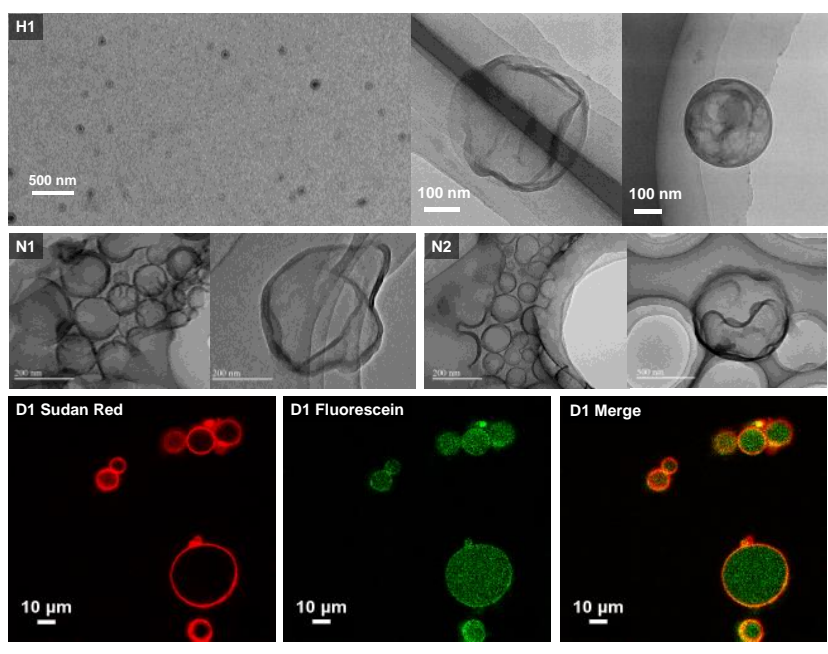

Figure 3. Transmission electron microscopy and fluorescence microscopy images of free-dried CNVs prepared from different methods (hydration, H1; nanoprecipitation, N1 and N2; dialysis, D1).

At a stock concentration of $0.1 \mathrm{mg} / \mathrm{mL}$, the diameters of the two CNVs (N1 and N2) showed only slight increase after 10 days with or without BSA $(0.4 \mathrm{mg} / \mathrm{mL}$ ) presence (Figure S6, the SEM images suggest that the proteins may be partially integrated into the CNVs from the nanosized holes on the membranes), while PLA made from ethylene glycol as the initiator could not form stable nanosuspension at all in PBS or with BSA using the same protocol: bulk aggregates were noted immediately following the nanoprecipitation method, suggesting the key to stability in physiological condition is the zwitterionic "lipid head". Polyethylene glycol (PEG) is a flexible, hydrophilic polymer that is widely used to modify nanovesicle to reduce protein binding and renal clearance. However, animal studies also indicate that the administration of some PEGylated drugs results in the generation of antibodies that specifically bind to PEG and reduce treatment efficacy or cause adverse drug reactions. ${ }^{[15]}$ The increasing realization that anti-PEG antibodies may have clinical impact is reflected in the United States Food and Drug Administration calling for measurement of anti-PEG antibody responses in new drugs that incorporate PEG molecules. ${ }^{[16]}$ Many normal individuals also have pre-existing antibodies against PEG in their circulation, likely due to the widespread use of PEG in many cosmetic and healthcare products. ${ }^{[17]}$ Therefore, the current zwitterionic CNV systems can be promising candidates for delivery in vivo.

Next, the acute cytotoxicity was tested using Hela cell line with the standard MTT essay (Figure 4b), from which the cell viability in the presence of either $\mathbf{N} 1$ or $\mathbf{N} 2 \mathrm{CNVs}(0.04 \mathrm{mg} / \mathrm{mL})$ maintains high after $4 \mathrm{~h}$. The same CNV condition was used for in vitro delivery throughout the studies. For the initial trial of in vitro delivery experiments, aqueous solutions of hydrophilic molecules such as PEGylated fluorescein isothiocyanate ([PEG-FITC] $=2.0$ $\mu \mathrm{M})$, PEGylated rhodamine $\mathrm{B}([\mathrm{PEG}-\mathrm{RhB}]=2.0 \mu \mathrm{M})$, and anticancer drug doxorubicin hydrochloride $([D O X]=20 \mu \mathrm{M})$ were encapsulated inside CNVs via the nanoprecipitation method (Figure S7-S9, SI). The average hydrodynamic diameters were examined for the CNVs loaded with various molecules (Figure 5c); however, no substantial difference in size was noted between CNVs fabricated in water vs. any other solution. Using PEGlyated FITC for the cellular update experiment is a "double-safety" feature since the dye is incapable of entering cells without delivery vehicles. Furthermore, to exclude the possibility of kinetically trapped (e.g., surface-adsorbed) dye molecules which may be carried into cells along with the CNVs (which may potentially interfere with capsulated dyes in fluorescence microscopy), the CNVs $(1.0 \mathrm{~mL})$ were dialyzed against a copious amount of water $(200 \mathrm{~mL})$ to ensure that no equilibrium of absolute dye concentrations inside and outside of the CNV membranes could be reached, and the fluorescence intensity of the aqueous solution outside the dialysis membrane was monitored over time (Figure 4c and Figure S10). After one week, the CV nanosuspensions inside the membrane remained orange (PEGFITC) and magenta (PEG-RhB), while the aqueous solution outside the dialysis bag was colorless to the naked eye, suggesting high CNV stability vs. liposomes. It can be seen from the emission intensity curves that 1) dye molecules ceased to accumulate in the extra-membrane milieu of the CNVs approximately $48 \mathrm{~h}$ after dialysis, and 2) dye-laden CNVs fabricated from high-MW $\alpha$ GPC-PLA (N2) exhibited consistently better dye entrapments, in that less fluorescence intensity was detected in each case compared to N1. The HeLa cells were first incubated with free PEG-FITC and PEG-RhB solutions at a dye concentration of $0.4 \mu \mathrm{M}$ for $4 \mathrm{~h}$. For PEG-FITC (Figure $4 \mathrm{e}$ ), no fluorescence emission characteristic of FITC could be received, indicating that PEGylation prevents the fluorescein derivative from entering live cells. However, PEG-RhB appeared to have mostly lightened up the endoplasmic reticulum regions under the same condition presumably due to the positive charge of dye and appropriate molecular weight (Figure 4f). ${ }^{[18]}$ In comparison, incubation with CNVs loaded with FITC-PEG with the same concentration (calibrated with UV-vis absorption spectroscopy, Figure S7-S9) led to strong FITC fluorescence signal in the perinuclear areas for most cells (Figure $4 \mathrm{~g}$ and $4 \mathrm{~h}$ ) and in the nucleus for certain cells, which appeared to be going through mitotic divisions (indicated by the arrow in Figure 4g). Although the average diameter for $\mathbf{N 1}$ is nearly twice that of $\mathbf{N 2}$, no observable difference in the fluorescence localization could be distinguished between PEG-FITC@N1 and PEG-FITC@N2. For cells transfected with PEG-RhB@N1 and PEG-RhB@N2 at 4 h, the rhodamine $B$ fluorescence signal was more widely disseminated within the cell plasma with no obvious preferential loci of organelles (Figure $4 \mathrm{i}$ and $4 \mathrm{j}$ ). After $24 \mathrm{~h}$, all cells that have taken up these dye-laden CNVs appear normal with no apparent signs of stress, lesion or apoptosis. The experiment shows that the N1 and N2 systems indeed exhibit in vitro biocompatibility. 
a)
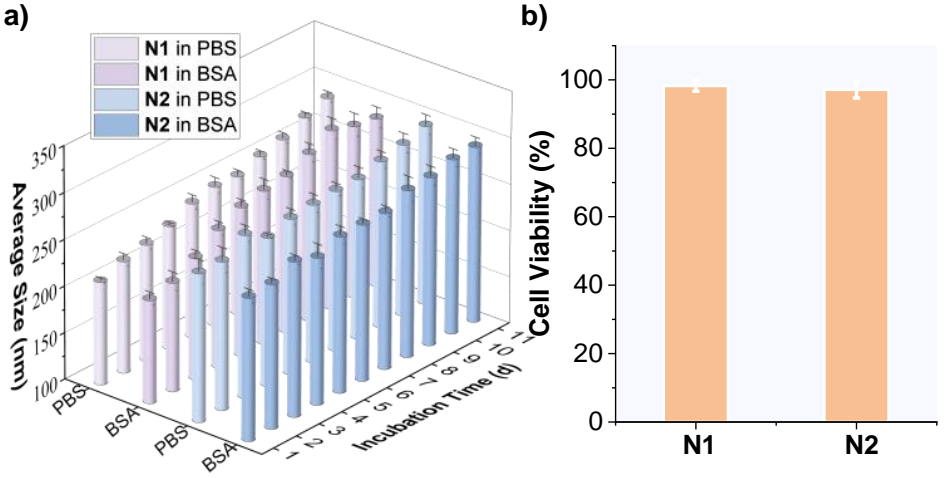

d)
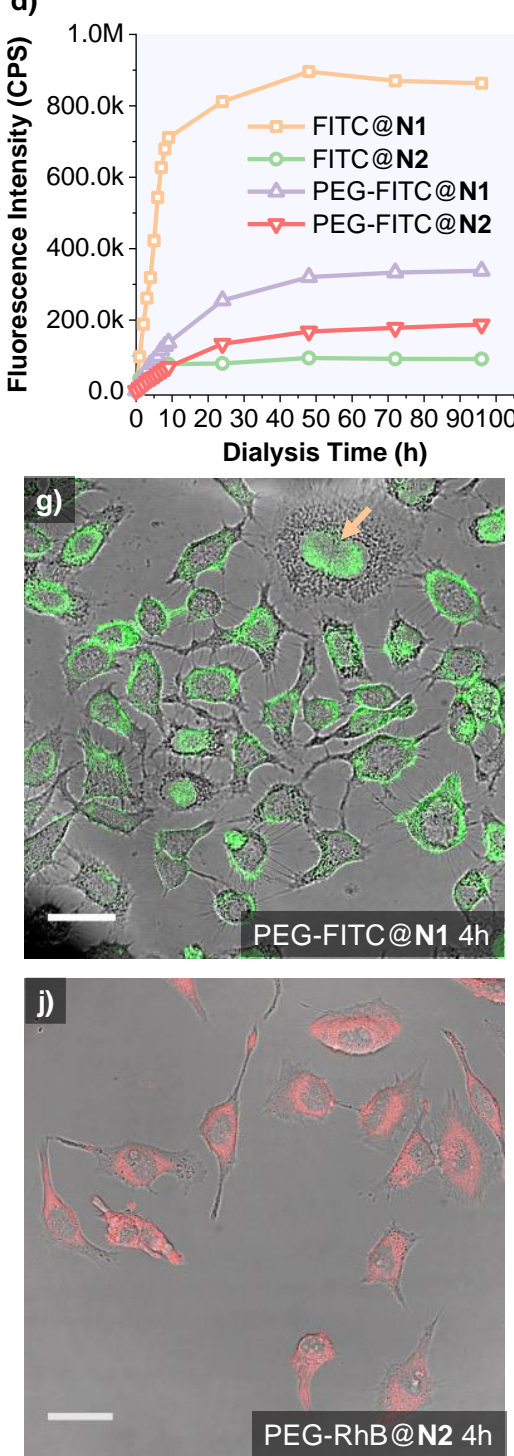
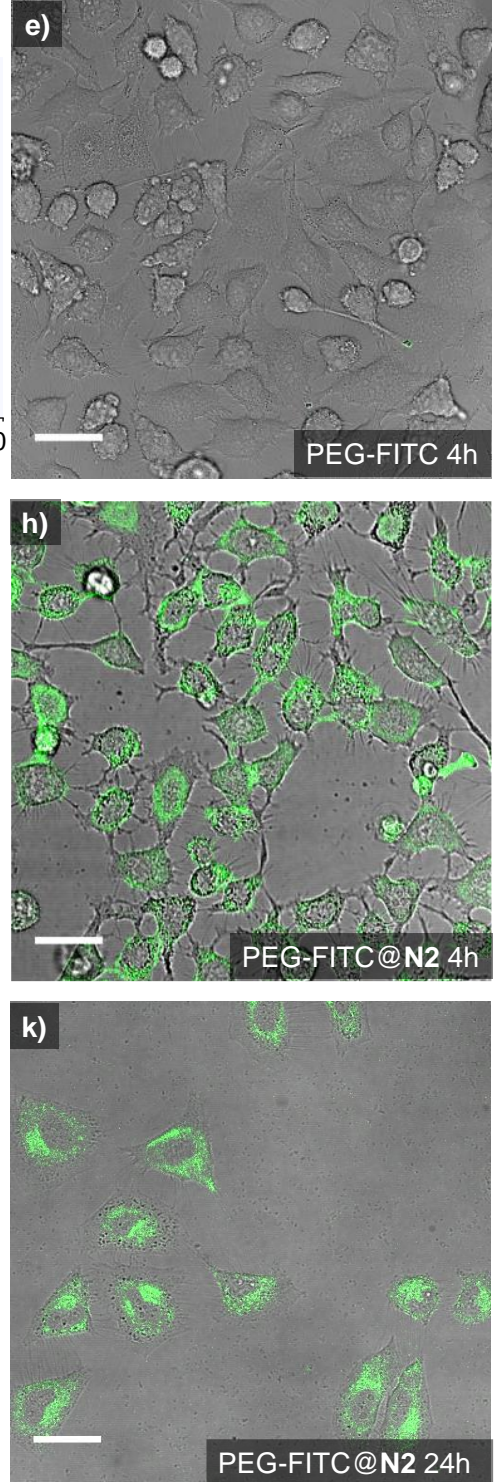

c)
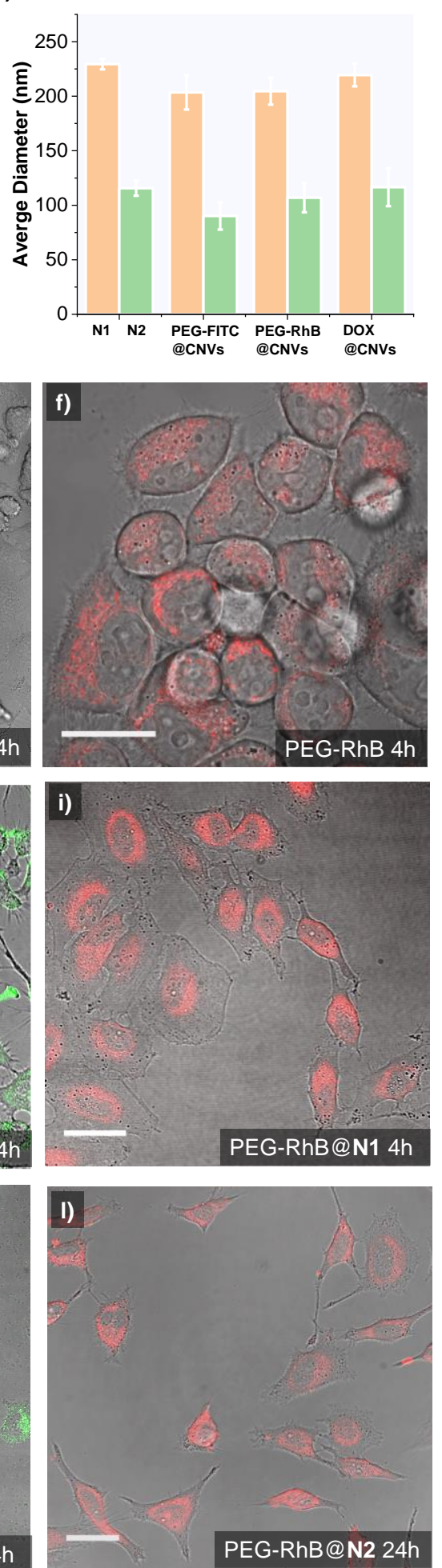

Figure 4. (a) Changes of average sizes (in hydrodynamic diameter) for CNVs N1 and N2 fabricated in PBS and in PBS in the presence of BSA (0.4 mg/mL) solutions over the course of 10 days monitored by DLS. (b) Cell viability rate after $4 \mathrm{~h}$ using the standard MTT essay in the presence of CNVs N1 and N2 at the same concentration of $0.04 \mathrm{mg} / \mathrm{mL}$. (c) Average diameters for CNVs co-fabricated with PEG-FITC (PEG-FITC@N1 and PEG-FITC@N2), PEG-RhB (PEGRhB@N1 and PEG-RhB@N2), and DOX (PEG-DOX@N1 and PEG-DOX@N2), respectively (orange bar: N1; green bar: N2). (d)Time-dependent fluorescence intensity (monitored at $520 \mathrm{~nm}$ ) change for the extra-membrane ultrapure-water used for dye-laden CNVs dialysis. (e)-(l) Merged bright-field and fluorescence confocal microscopy images of HeLa cells incubated in the presence of different agents at $37 \mathrm{C}$ in a $95 \%-\mathrm{CO}_{2}$ atmosphere: (e) PEG-FITC solution (0.4 $\left.\mu \mathrm{M}\right)$ for 4 h; (f) PEG-RhB solution (0.4 $\mu \mathrm{M}$ ) for 4 h; (g) PEG-FITC@N1 for 4 h; (h) PEG-FITC@N2 for 4 h; (i) PEG-RhB@N1 for 4 h; (j) PEG-RhB@N2 for 4 h; (g) PEG-FITC@N2 for 24 h; (h) PEG-RhB@N2 for 24 h. Scale bar: $20 \mu \mathrm{m}$. Green channel: $\lambda_{\mathrm{ex}}=488 \mathrm{~nm} ; \lambda_{\mathrm{em}}=495-600 \mathrm{~nm}$; red channel: $\lambda_{\mathrm{ex}}=543 \mathrm{~nm}$; $\lambda_{\mathrm{em}}=$ $555-700 \mathrm{~nm}$. 


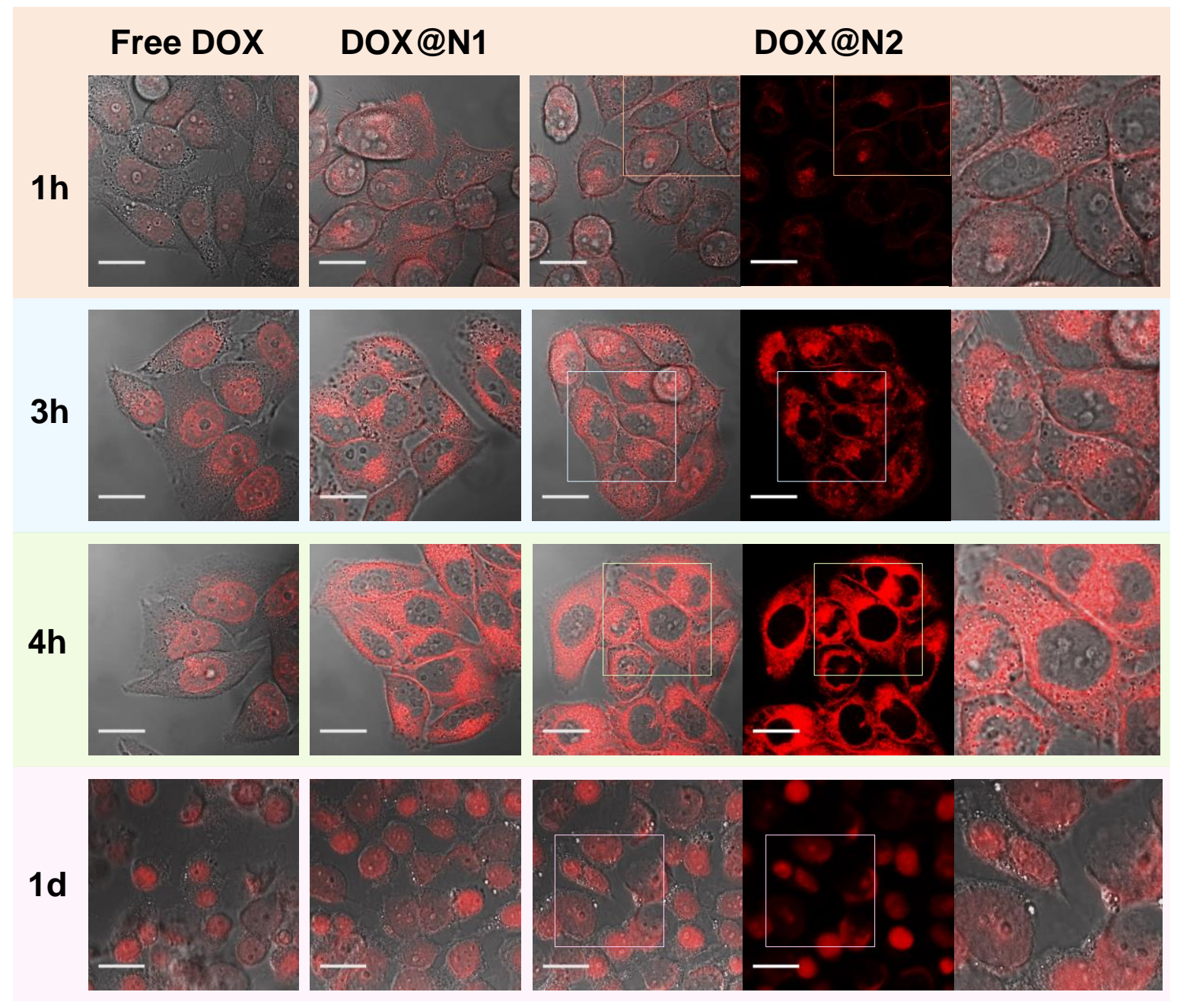

Figure 5. Confocal fluorescence microscopy images of HeLa cells incubated with doxorubicin hydrochloride solution and CNVs loaded with doxorubicin hydrochloride solution (DOX@N1 and DOX@N2) taken at various time points. $\left(\lambda_{\mathrm{ex}}=488 \mathrm{~nm}, \lambda_{\mathrm{em}}=535-650 \mathrm{~nm}\right.$ ). Scale bar: 20 $\mu \mathrm{m}$.

Finally, to test whether these CNVs can release bioactive molecules into cells, DOX@N1 and DOX@N2 were used in a comparative study with the free DOX solution at the same drug concentration calibrated with UV-vis spectroscopy (Figure 5). For the free DOX, the fluorescence signal from DOX is largely confined in the nucleus throughout the experiment, presumably due to DOX's ability for ds-DNA intercalation. When DOX molecules were loaded into CNVs N1 and N2, the fluorescence signal after incubation for $1 \mathrm{~h}$ was more pronounced around the Golgi apparatus. However, as time elapsed, the fluorescence intensity became gradually evenly distributed on all membrane structures within the cells. After $3 \mathrm{~h}$ and $4 \mathrm{~h}$, the fluorescence intensity from the DOX@N1 and DOX@N2 outweighed that from the free DOX control group at the same experimental setup, suggesting higher doxorubicin uptake by cells using the CNVs as delivery vehicles. Regardless of the drastically different accumulation loci of the CNVs vs. free doxorubicin molecules, cell deaths were observed for all groups, although the dynamics and kinetics of the drug release processes are yet to be investigated. Nonetheless, the current study has pointed to the possibility for further in vivo studies as clinical drug carriers in the next stages.

\section{Conclusion}

In conclusion, inspired by the molecular structures constituting natural liposomes and synthetic polymersomes, we combined the chemical features of the two systems into one by the $\alpha \mathrm{GPC}$ initiated ring-opening polymerization (ROP) of L-lactide. The two naturally occurring moieties are covalently tethered together by chemical synthesis to yield a lipid-head-polymer-tail structure. It was found that the chimeric structure is capable of forming nanovesicles (CNVs) in water via three different types of methods (hydration, nanoprecipitation, and dialysis). The morphologies of the CNVs are characterized by DLS, SEM, TEM and confocal FLM: it was determined that nanoprecipitation was the preferred method for CNVs fabrication. The CNVs prepared from the zwitterionic polymers remain stable in PBS in the presence of proteins. Various molecules including fluorescent dyes and doxorubicin aqueous solutions were loaded into these CNVs, which were then used to incubate with HeLa cells. It was found that the CNVs could accumulate inside the cells and were tested as drug delivery vehicles: the CNVs loaded with doxorubicin were demonstrated to have induced cell deaths after $24 \mathrm{~h}$, while CNVs loaded with fluorescent dyes were not toxic to cells. Notably, the facile ROP method allows for laboratory synthesis on a scale of 500 grams, which is a substantial advantage over many other 
block-copolymer based polymersomes in terms of clinical translation. Given the large variety of lipid-head structures available, we expect that the current example is only the first one of many such CNV systems to design and synthesize in the future.

\section{Experimental Section}

Materials. L- $\alpha$ glycerylphosphorylcholine ( $\alpha \mathrm{GPC}), \mathrm{Sn}(\mathrm{Oct})_{2}$, L-lactide , 3 (4,5-Dimethylthiazol-2-yl)-2,5-diphenyltetrazolium bromide (MTT), PEGFITC (5 kDa), PEG-rhodamine b $(5 \mathrm{kDa})$, and $\mathrm{DOX} \cdot \mathrm{HCl}$ were purchased from Shanghai Aladdin Biochemical Technology Co.,Ltd and were used without any purification. Bovine serum albumin was purchased from Zhejiang Tianhang Biotechnology Co., Ltd. The Dulbecco's modified Eagle medium (DMEM) and trypsin were purchased from Meilun Biotechnology Co., Ltd. Dalian. Instruments. Proton nuclear magnetic resonance $\left({ }^{1} \mathrm{H}\right.$ NMR) spectra were recorded at $25{ }^{\circ} \mathrm{C}$ on a Bruker AV300 NMR spectrometer $(300 \mathrm{MHz})$ with tetramethylsilane as the internal standard and $\mathrm{CDCl}_{3}$ as the solvent. Fourier transform infrared (FTIR) spectra were recorded on a Bruker VECTOR-22 spectrometer in $\mathrm{KBr}$ pellets with the $4000-500 \mathrm{~cm}^{-1}$ range, at a $4-\mathrm{cm}^{-1}$ scanning frequency. Molecular weights and distributions were determined by gel-permeation chromatography (Agilent 1260, DMF, $1.0 \mathrm{~mL} / \mathrm{min}$ ) vs. polystyrene standards with RI detection. Nano rook 90 -Plus was used to measure the size distributions and zeta potentials of the vesicles. Size and zeta potential values were determined by three and five repeated measurements at $25^{\circ} \mathrm{C}$ for each sample. UV/Vis absorption spectra were recorded on a PerkinEImer Lambda 465 UV-Vis spectrometer. Transmission electron microscopy (TEM, TECNAI T12 120KV), high-resolution transmission electron microscopy (HRTEM, Talos F200X) and scanning electron microscope (SEM, GeminiSEM 500) were conducted to observe the morphology and diameters of vesicles. The vesicle solutions were dropped on copper meshes and dried at room temperature for TEM and HRTEM. The lyophilized powder of vesicle solution was treated by spray-gold for SEM. Polymer synthesis. $\alpha$ GPC-PLA was synthesized by direct condensation from $L-\alpha$ glycerylphosphorylcholine and L-lactide with molar ratios of $1 / 30$ and $1 / 50$. Into a thoroughly dried 2-L Schlenk flask with a magnetic stirrer, $\alpha$ GPC (29.8 or $17.8 \mathrm{~g}$ ) and L-lactide $(500 \mathrm{~g})$ were placed. Before heating, pumping vacuum and purging with nitrogen gas to ensure the Schlenk flask is completely free of oxygen and water. $\mathrm{Sn}(\mathrm{Oct})_{2}(0.04 \%)$ was added into melting GPC and L-lactide under nitrogen protection. The reaction was heated for $24 \mathrm{~h}$ and cooled down to room temperature to obtain the $\alpha \mathrm{GPC}$ PLA polymer. The polymer was dissolved in dichloromethane and purified by reprecipitation in ethanol twice and diethyl ether once, and was then dried under vacuum at $40{ }^{\circ} \mathrm{C}(380 \mathrm{~g}$ or $412 \mathrm{~g})$. Vesicle preparation by hydration. The $\alpha$ GPC-PLA polymer $\left(2.0 \mathrm{mg}\right.$ ) was dissolved in $\mathrm{CH}_{2} \mathrm{Cl}_{2}$ (1 $\mathrm{mL}$ ) and was dried udner nitrogen to form a thin polymer film desiccated under vacuum overnight. The polymer film was rehydrated in ultrapure water $(10 \mathrm{~mL})$ at $37^{\circ} \mathrm{C}$ for overnight. The resulting solutions was sonicated for $0.5 \mathrm{~h}$ and subjected to five freeze-thaw cycles. Vesicle preparation by nanoprecitation. The $\alpha$ GPC-PLA polymer $(20 \mathrm{mg})$ was dissolved in THF (10 mL) to form a homogeous solution, which was then slowly added into ultrapure water $(100 \mathrm{~mL})$ at a constant speed during 20 min under vigorous magnetic stirring. The organic solvent was removed by stirring over night. The mixture solution was dialyzed against ultrapure water $(2 \mathrm{~L})$ for $48 \mathrm{~h}$. Vesicle preparation by dialysis. Florescence labeled giant vesicles were prepared by dialysis. The $\alpha$ GPC-PLA polymer $(2.0 \mathrm{mg})$, Sudan Red III $(20 \mu \mathrm{mol})$ and FITC-Na $(20 \mu \mathrm{mol})$ were dissolved in a $\mathrm{THF} /$ methanol mixture solvent $(\mathrm{THF} /$ methanol $=4 / 1 \mathrm{~V} / \mathrm{V}, 8 \mathrm{~mL} / 2 \mathrm{~mL})$. The mixture solution was transferred into a dialysis bag (MWCO:1 kDa) and was dialyzed against ultrapure water $(2 \mathrm{~L})$ for $48 \mathrm{~h}$. Drug-loaded vesicle preparation by nanoprecitation. PEG-florescence $(0.1 \mu \mathrm{mol})$ or $\mathrm{DOX} \cdot \mathrm{HCl}(0.1 \mu \mathrm{mol})$ was dissolved in ultrapure water $(50 \mathrm{~mL})$. The $\alpha \mathrm{GPC}$ PLA polymer $(10 \mathrm{mg})$ was dissolved in THF $(10 \mathrm{~mL})$ to form a homogeous solution, which was then slowly added into ultrapure water $(50 \mathrm{~mL})$ at a constant speed over 20 min under rigorous magnetic stirring. The organic solvent was removed by stirring over night. The mixture solution was dialyzed against ultrapure water for 48 h. Fluorescence dye de- adsorption release experiment. PEGylated fluorescent dye-loaded CNV solutions were transferred into dialysis bags (MWCO: $7 \mathrm{kDa}$ ) and were dialyzed in ultrapure water $(200 \mathrm{~mL})$. The aqoueous solution $(1 \mathrm{~mL})$ outside the dialysis bag was taken out for fluorescence measurements and fresh ultrapure water $(1 \mathrm{~mL})$ was added back to keep the volume constant. Confocal laser scanning microscopy (CLSM). Under a humidified atmosphere containing $5 \% \mathrm{CO}_{2}$, HeLa cells were cultured in a confocal microscope dish in DMEM containing FBS (10\%) and penicillinstreptomycin (1\%) at $37^{\circ} \mathrm{C}$ for $24 \mathrm{~h}$. Cells were first treated with the CNV solution $(0.04 \mathrm{mg} / \mathrm{mL}$ by polymer weight content), washed with PBS $(3 \times)$ and was then imaged by a CLSM (Zeiss LSM880). Acute cytotoxicity. The acute cytotoxicity assay was performed by an MTT assay agains HeLa cells. The HeLa cells were seeded in a 96-well culture plate $(10,000$ cells/well) with DMEM $(100 \mu \mathrm{L})$ for $24 \mathrm{~h}$, and were exposed to conjugates with or without the CNV solution $(0.04 \mathrm{mg} / \mathrm{mL}$ by polymer weight content) for another $4 \mathrm{~h}$. The DMEM medium was subsequently removed and DMEM containing MTT $(5 \mathrm{mg} / \mathrm{mL})$ was added to each well for further incubation for $4 \mathrm{~h}$. The concentration of the proliferating cells in each well was confirmed using a microplate reader at the test wavelength of $570 \mathrm{~nm}$. The values are expressed as mean $\pm \operatorname{SD}(n=3)$. The relative cell viability is calculated according to the following equation: Cell viability $(\%)=$ $\left(A_{\text {samples }}-A_{0}\right) /\left(A_{\text {control }}-A_{0}\right) \times 100$, where $A_{\text {samples }}$ and $A_{\text {control }}$ were obtained with or without CNV solutions, respectively, and $A_{0}$ was obtained with complete DMEM.

\section{Acknowledgements}

We thank the National Natural Science Foundation of China (21975238) for financial support.

Keywords: liposomes $\cdot$ polymersome $\cdot$ nanomedicine $\bullet$ nanovesicle $\cdot$ drug delivery

[1] a) E. Nance, S. H. Pun, R. Saigal, D. L. Sellers, Nat. Rev. Mater 2021, DOI: 10.1038/s41578-021-00394-w; b) Y. Barenholz, J. Control Release 2012, 160, 117-134; c) F. Wang, J. Xiao, S Chen, H. Sun, B. Yang, J. Jiang, X. Zhou, J. Du, Adv. Mater. 2018, 30, e1705674.

[2] a) J. P. Bost, H. Barriga, M. N. Holme, A. Gallud, M. Maugeri, D. Gupta, T. Lehto, H. Valadi, E. K. Esbjorner, M. M. Stevens, S. El-Andaloussi, ACS Nano 2021, 15, 13993-14021; b) X. Qin, C. Yu, J. Wei, L. Li, C. Zhang, Q. Wu, J. Liu, S. Q. Yao, W. Huang, Adv. Mater. 2019, 31, e1902791; c) S. Iqbal, M. Blenner, A. Alexander-Bryant, J. Larsen, Biomacromolecules 2020, 21 1327-1350; d) S. Lv, M. Sylvestre, A. N. Prossnitz, L. F. Yang, S. H. Pun, Chem. Rev. 2021, 121, 11653-11698; e) M Machtakova, H. Therien-Aubin, K. Landfester, Chem. Soc. Rev. 2021, DOI: $10.1039 /$ d1cs00686j.

[3] a) M. T. Manzari, Y. Shamay, H. Kiguchi, N. Rosen, M. Scaltriti, D. A. Heller, Nat. Rev. Mater. 2021, 6, 351-370; b) J. Wolfram, M. Ferrari, Nano Today 2019, 25, 85-98; c) Y. Zhu, B. Yang, S. Chen, J. Du, Prog. Polym. Sci. 2017, 64, 1-22.

[4] a) M. D. Shin, S. Shukla, Y. H. Chung, V. Beiss, S. K. Chan, O. A. Ortega-Rivera, D. M. Wirth, A. Chen, M. Sack, J. K. Pokorski N. F. Steinmetz, Nat. Nanotechnol. 2020, 15, 646-655; b) R. Tenchov, R. Bird, A. E. Curtze, Q. Zhou, ACS Nano 2021, DOI: 10.1021/acsnano.1c04996.

[5] W. T. Al-Jamal, K. Kostarelos, Acc. Chem. Res. 2011, 44, 1094 1104

[6] a) D. E. Discher, A. Eisenberg, Science 2002, 297, 967-973; b) Y. Y. Mai, A. Eisenberg, Chem. Soc. Rev. 2012, 41, 5969-5985.

[7] a) E. Rideau, R. Dimova, P. Schwille, F. R. Wurm, K. Landfester Chem. Soc. Rev. 2018, 47, 8572-8610; b) N. Grimaldi, F. Andrade, N. Segovia, L. Ferrer-Tasies, S. Sala, J. Veciana, N. Ventosa, Chem. Soc. Rev. 2016, 45, 6520-6545.

[8] a) F. Araste, A. Aliabadi, K. Abnous, S. M. Taghdisi, M. Ramezani, M. Alibolandi, J. Control Release 2021, 330, 502528; b) X. Wang, C. Yao, G. Zhang, S. Liu, Nat. Commun. 2020, 11,1524 ; c) B. M. Chen, T. L. Cheng, S. R. Roffler, ACS Nano 
2021, 15, 14022-14048; d) L. Maghak, J. Larsen, ACS Biomater. Sci. Eng. 2020, 6, 4846-4850; e) A. Albanese, P. S. Tang, W. C. Chan, Annu. Rev. Biomed. Eng. 2012, 14, 1-16; f) S. Matoori, J.-C. Leroux, Mater. Horizons 2020, 7, 1297-1309.

[9] S. Khan, J. McCabe, K. Hill, P. A. Beales, J. Colloid. Interface Sci. 2020, 562, 418-428.

[10] a) A. Michalski, M. Brzezinski, G. Lapienis, T. Biela, Prog. Polym. Sci. 2019, 89, 159-212; b) X. He, Y. He, M. S. Hsiao, R L. Harniman, S. Pearce, M. A. Winnik, I. Manners, J. Am. Chem. Soc. 2017, 139, 9221-9228; c) H. T. H. Nguyen, G. N. Short, P. Qi, S. A. Miller, Green Chem. 2017, 19, 1877-1888.

[11] a) G. Zhang, J. Chen, S. J. Payne, S. E. Kooi, J. N. Demas, C. L. Fraser, J. Am. Chem. Soc. 2007, 129, 8942-8943; b) G. Zhang, R. E. Evans, K. A. Campbell, C. L. Fraser, Macromolecules 2009, 42, 8627-8633.

[12] a) Z. Liu, J. Cui, W. Zhan, Langmuir 2020, 36, 13193-13200; b) J. P. Reeves, R. M. Dowben, j. Cell. Physiol. 1969, 73, 49-60 c) M. Ismail, L. Ling, Y. Du, C. Yao, X. Li, Biomaterials 2018 $163,76-87$; d) G. Liu, S. Ma, S. Li, R. Cheng, F. Meng, H. Liu, Z. Zhong, Biomaterials 2010, 31, 7575-7585.

[13] a) J. M. H. Kremer, M. W. Van der Esker, C. Pathmamanoharan, P. H. Wiersema, Biochemistry 1977, 16, 3932-3935; b) D. Zhang, Y. Fan, H. Chen, S. Trépout, M.-H. Li, Angew. Chem. Int. Edit. 2019, 58, 10260-10265; c) N. Zhang, H. Chen, Y. Fan, L. Zhou, S. Trepout, J. Guo, M. H. Li, ACS Nano 2018, 12, 40254035.

[14] a) Y. Peng, X. Zhu, L. Qiu, Biomaterials 2016, 106, 1-12; b) R. Ridolfo, D. S. Williams, J. C. M. van Hest, Polym. Chem. 2020, $11,2775-2780$

[15] a) Y. Liu, C. A. Smith, J. C. Panetta, W. Yang, L. E. Thompson, J. P. Counts, A. R. Molinelli, D. Pei, N. M. Kornegay, K. R. Crews, H. Swanson, C. Cheng, S. E. Karol, W. E. Evans, H. Inaba, C.-H. Pui, S. Jeha, M. V. Relling, J. Clin. Oncol. 2019, 37 , 2051-2061; b) J. K. Armstrong, G. Hempel, S. Koling, L. S. Chan T. Fisher, H. J. Meiselman, G. Garratty, Cancer 2007, 110, 103111 ; c) M. S. Hershfield, N. J. Ganson, S. J. Kelly, E. L. Scarlett, D. A. Jaggers, J. S. Sundy, Arthritis Res. Ther. 2014, 16, R63.

[16] J. J. F. Verhoef, J. F. Carpenter, T. J. Anchordoquy, H. Schellekens, Drug Discov. Today 2014, 19, 1945-1952.

[17] Q. Yang, S. K. Lai, WIREs Nanomed. Nanobiotechnol. 2015, 7, 655-677.

[18] a) P. Reungpatthanaphong, S. Dechsupa, J. Meesungnoen, C. Loetchutinat, S. Mankhetkorn, J. Biochem. Biophys. Methods 2003, 57, 1-16; b) N. Morimoto, R. Takei, M. Wakamura, Y. Oishi, M. Nakayama, M. Suzuki, M. Yamamoto, F. M. Winnik, Sci. Rep. 2018, 8, 1128 . 


\section{Entry for the Table of Contents}

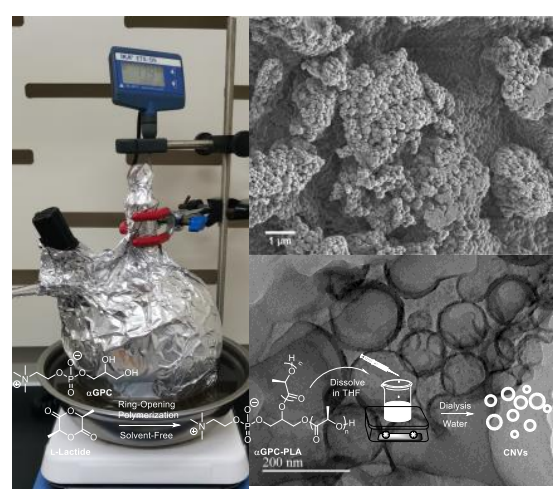

A "lipid-head-polymer-tail" structure could be created by covalently linking bio-derived molecular building blocks together, via solventfree ring-opening polymerization at a 500-g scale. The "chimeric" nanovesicle system is readily obtained in aqueous environments with combined benefits from both liposomes and polymersomes, and is a promising alternative delivery platform for nanomedicine. 